\title{
Asymptotic behavior of reciprocal sum of two products of Fibonacci numbers
}

Ho-Hyeong Lee ${ }^{1}$ and Jong-Do Park ${ }^{1 *}$

${ }^{\text {"Correspondence: }}$

mathjdpark@khu.ac.kr

${ }^{1}$ Department of Mathematics and Research Institute for Basic Sciences, Kyung Hee University, Seoul, Korea

\section{Abstract}

Let $\left\{f_{k}\right\}_{k=1}^{\infty}$ be a Fibonacci sequence with $f_{1}=f_{2}=1$. In this paper, we find a simple form $g_{n}$ such that

$$
\lim _{n \rightarrow \infty}\left\{\left(\sum_{k=n}^{\infty} a_{k}\right)^{-1}-g_{n}\right\}=0
$$

where $a_{k}=\frac{1}{f_{k}^{2}}, \frac{1}{f_{k} f_{k+m}}$, or $\frac{1}{f_{3 k}^{2}}$. For example, we show that

$$
\lim _{n \rightarrow \infty}\left\{\left(\sum_{k=n}^{\infty} \frac{1}{f_{3 k}^{2}}\right)^{-1}-\left(f_{3 n}^{2}-f_{3 n-3}^{2}+\frac{4}{9}(-1)^{n}\right)\right\}=0 .
$$

MSC: 11B39; 11Y55; 40A05

Keywords: Fibonacci number; Reciprocal sum; Catalan's identity; Convergent series

\section{Introduction}

Last decade many mathematicians were interested in finding the formula for the integer part of the reciprocal tails of the convergent series. Precisely, one can see the explicit value of $\left\lfloor\left(\sum_{k=n}^{\infty} a_{k}\right)^{-1}\right\rfloor$ when $\sum_{k=1}^{\infty} a_{k}$ converges. This problem starts from the reciprocal sum of Fibonacci numbers. Let $f_{0}=0, f_{1}=f_{2}=1$, and $f_{n+2}=f_{n}+f_{n+1}$ for any $n \in \mathbb{N}$. In [6], Ohtsuka and Nakamura proved

$$
\left\lfloor\left(\sum_{k=n}^{\infty} \frac{1}{f_{k}}\right)^{-1}\right\rfloor= \begin{cases}f_{n-2}, & n \geq 2 \text { is even } \\ f_{n-2}-1, & n \geq 1 \text { is odd }\end{cases}
$$

and

$$
\left\lfloor\left(\sum_{k=n}^{\infty} \frac{1}{f_{k}^{2}}\right)^{-1}\right\rfloor= \begin{cases}f_{n-1} f_{n}-1, & n \geq 2 \text { is even; } \\ f_{n-1} f_{n}, & n \geq 1 \text { is odd }\end{cases}
$$

(c) The Author(s) 2020. This article is licensed under a Creative Commons Attribution 4.0 International License, which permits use, sharing, adaptation, distribution and reproduction in any medium or format, as long as you give appropriate credit to the original author(s) and the source, provide a link to the Creative Commons licence, and indicate if changes were made. The images or other third party material in this article are included in the article's Creative Commons licence, unless indicated otherwise in a credit line to the material. If material is not included in the article's Creative Commons licence and your intended use is not permitted by statutory regulation or exceeds the permitted use, you will need to obtain permission directly from the copyright holder. To view a copy of this licence, visit http://creativecommons.org/licenses/by/4.0/. 
where $\lfloor x\rfloor$ is the floor function. See more results for subsequences of Fibonacci numbers in $[11,12]$, Pell numbers in $[1,13]$, and Mathieu series in [4]. Also, recently many interesting results on special numbers have been obtained in [7-9]. The following natural question on the asymptotic behavior can be raised.

Question Let $\sum_{k=1}^{\infty} a_{k}$ be a convergent series. Can we find a suitable function $g_{n}$ such that

$$
\left(\sum_{k=n}^{\infty} a_{k}\right)^{-1} \sim g_{n} ?
$$

Here the notation $A_{n} \sim B_{n}$ means that $\lim _{n \rightarrow \infty}\left(A_{n}-B_{n}\right)=0$.

In [3], we proved that

$$
\left(\sum_{k=n}^{\infty} \frac{1}{f_{m k-\ell}}\right)^{-1} \sim f_{m n-\ell}-f_{m(n-1)-\ell}
$$

for any $m \in \mathbb{N}$ and $0 \leq \ell \leq m-1$. In fact, we proved

$$
f_{m n-\ell}-f_{m(n-1)-\ell}-\frac{1}{f_{n}}<\left(\sum_{k=n}^{\infty} \frac{1}{f_{m k-\ell}}\right)^{-1}<f_{m n-\ell}-f_{m(n-1)-\ell}+\frac{1}{f_{n}} .
$$

In the special case when $m=1$ and $\ell=0$, the above equation is reduced to

$$
\lim _{n \rightarrow \infty}\left\{\left(\sum_{k=n}^{\infty} \frac{1}{f_{k}}\right)^{-1}-f_{n-2}\right\}=0 .
$$

In [3], we also proved the generalization of (1.1) as the following formula:

$$
\left\lfloor\left(\sum_{k=n}^{\infty} \frac{1}{f_{m k-\ell}}\right)^{-1}\right\rfloor= \begin{cases}f_{m n-\ell}-f_{m(n-1)-\ell}-1, & m(n+1)+\ell \text { is even; } \\ f_{m n-\ell}-f_{m(n-1)-\ell}, & m(n+1)+\ell \text { is odd }\end{cases}
$$

for any $m \in \mathbb{N}$ and $0 \leq \ell \leq m-1$. One can see the results for the product of two Fibonacci numbers in [5].

In this paper, we study the asymptotic behavior of the reciprocal sum of

$$
f_{k}^{2}, f_{k} f_{k+m}, f_{3 k}^{2}
$$

for $k, m \in \mathbb{N}$. Precisely, we prove that

$$
\begin{aligned}
& \left(\sum_{k=n}^{\infty} \frac{1}{f_{k} f_{k+2 \ell}}\right)^{-1} \sim g_{n, \ell}, \quad \ell=0,1,2, \ldots \\
& \left(\sum_{k=n}^{\infty} \frac{1}{f_{k} f_{k+2 \ell-1}}\right)^{-1} \sim h_{n, \ell}, \quad \ell=1,2,3, \ldots
\end{aligned}
$$


where

$$
\begin{aligned}
& g_{n, \ell}:=f_{n+\ell-1} f_{n+\ell}-\left(f_{\ell}^{2}+(-1)^{\ell}\right) \frac{(-1)^{n}}{3}, \\
& h_{n, \ell}:=f_{n+\ell-1}^{2}-\left(f_{\ell-1} f_{\ell}+(-1)^{\ell}\right) \frac{(-1)^{n}}{3} .
\end{aligned}
$$

For the proof of our main theorem, we prove the following inequalities:

(i) $g_{n, 0}<\left(\sum_{k=n}^{\infty} \frac{1}{f_{k}^{2}}\right)^{-1}<g_{n, 0}+c_{n}$ for all $n \geq 1$,

(ii) $g_{n, \ell}-c_{n}<\left(\sum_{k=n}^{\infty} \frac{1}{f_{k} f_{k+2 \ell}}\right)^{-1} \leq g_{n, \ell}$ for all $\ell \geq 1$ and $n \geq 2 \ell-1$,

(iii) $h_{n, \ell}-c_{n}<\left(\sum_{k=n}^{\infty} \frac{1}{f_{k} f_{k+2 \ell-1}}\right)^{-1}<h_{n, \ell}$ for all $\ell \geq 1$ and $n \geq 2 \ell-2$.

Here $c_{n}=1 / f_{n}$. We believe that the conditions $n \geq 2 \ell-1$ and $n \geq 2 \ell-2$ can be removed. However, it is enough to prove the inequalities for sufficiently large $n$ for the study of asymptotic behavior as $n \rightarrow \infty$. As an application of the above results, we can obtain

$$
\left\lfloor\left(\sum_{k=n}^{\infty} \frac{1}{f_{k} f_{k+m}}\right)^{-1}\right\rfloor
$$

for all $m \in \mathbb{N}$. For example, our formulas imply that

$$
\left\lfloor\left(\sum_{k=n}^{\infty} \frac{1}{f_{k} f_{k+8}}\right)^{-1}\right\rfloor= \begin{cases}f_{n+3} f_{n+4}-4, & n \text { is even; } \\ f_{n+3} f_{n+4}+3, & n \text { is odd }\end{cases}
$$

and

$$
\left\lfloor\left(\sum_{k=n}^{\infty} \frac{1}{f_{k} f_{k+7}}\right)^{-1}\right\rfloor= \begin{cases}f_{n+3}^{2}-3, & n \text { is even; } \\ f_{n+3}^{2}+2, & n \text { is odd }\end{cases}
$$

In the final section, we discuss the reciprocal sum of $f_{m k}^{2}$ for $m \geq 2$. If $m=3$, then we prove that

$$
\left(\sum_{k=n}^{\infty} \frac{1}{f_{3 k}^{2}}\right)^{-1} \sim \widetilde{g}_{n}
$$

where $\widetilde{g}_{n}=f_{3 n}^{2}-f_{3 n-3}^{2}+\frac{4}{9}(-1)^{n}$.

As in $[3,6]$, the following lemma plays an important role in proving the essential inequalities.

Lemma 1.1 ([6]) Let $\left\{a_{n}\right\}_{n=1}^{\infty}$ and $\left\{b_{n}\right\}_{n=1}^{\infty}$ be sequences with $\lim _{n \rightarrow \infty} a_{n}=0$. If $a_{n}<b_{n}+a_{n+1}$ holds for any $n \in \mathbb{N}$, then

$$
a_{n}<\sum_{k=n}^{\infty} b_{k}
$$

holds for any $n \in \mathbb{N}$.

We use the following relation when we calculate Fibonacci numbers. 
Lemma 1.2 ([2], Catalan's identity) For any $n, k \in \mathbb{N}$, we have

$$
f_{n}^{2}=f_{n+k} f_{n-k}+(-1)^{n+k} f_{k}^{2}
$$

The following lemma is useful when we get a lower bound of formulas containing Fibonacci numbers. It comes from the identity

$$
f_{m+n}=f_{m-1} f_{n}+f_{m} f_{n+1} .
$$

Lemma 1.3 For any $m, n \in \mathbb{N}$, we have $f_{m+n}>f_{m+1} f_{n}$.

Remark 1.4 The Fibonacci numbers $f_{n}$ can be written as the closed form by Binet's formula [10]

$$
f_{n}=\frac{\alpha^{n}-\beta^{n}}{\alpha-\beta}
$$

where $\alpha>\beta$ are two solutions of $x^{2}-x-1=0$. The infinite sum $\sum_{k=1}^{\infty} \frac{1}{f_{k}}$ is known to be irrational, but it is unknown whether $\sum_{k=1}^{\infty} \frac{1}{f_{k}^{2}}$ is irrational or not.

\section{Reciprocal sum of $f_{k}^{2}$}

In [6], Ohtsuka and Nakamura proved the following inequalities:

(i) $f_{n-1} f_{n}-1<\left(\sum_{k=n}^{\infty} \frac{1}{f_{k}^{2}}\right)^{-1}<f_{n-1} f_{n}$, when $n$ is even,

(ii) $f_{n-1} f_{n}<\left(\sum_{k=n}^{\infty} \frac{1}{f_{k}^{2}}\right)^{-1}<f_{n-1} f_{n}+1$, when $n$ is odd.

Now we will prove the inequalities which are sharper than (i) and (ii). In this section, let

$$
g_{n}=g_{n, 0}=f_{n-1} f_{n}-\frac{1}{3}(-1)^{n} .
$$

In fact, $g_{n}$ can be written as

$$
\begin{aligned}
g_{n} & =f_{n-1} f_{n}-\frac{1}{3}(-1)^{n} \\
& =\left\{f_{n-2} f_{n+1}+(-1)^{n}\right\}-\frac{1}{3}(-1)^{n} \\
& =f_{n}^{2}-f_{n-1}^{2}+\frac{2}{3}(-1)^{n} .
\end{aligned}
$$

Theorem 2.1 For any $n \in \mathbb{N}$, we have

$$
g_{n}<\left(\sum_{k=n}^{\infty} \frac{1}{f_{k}^{2}}\right)^{-1}<g_{n}+c_{n},
$$

where $c_{n}=1 / f_{n}$.

To prove Theorem 2.1, we need the following formula.

Proposition 2.2 For any $n \in \mathbb{N}$, we have

$$
\left(g_{n+1}-g_{n}\right) f_{n}^{2}-g_{n} g_{n+1}=\frac{1}{9} .
$$


Proof Note that

$$
\begin{aligned}
\left(g_{n+1}-g_{n}\right) f_{n}^{2} & =\left\{f_{n} f_{n+1}-f_{n-1} f_{n}+\frac{2}{3}(-1)^{n}\right\} f_{n}^{2} \\
& =f_{n}^{4}+\frac{2}{3}(-1)^{n} f_{n}^{2} .
\end{aligned}
$$

By Catalan's identity, we have

$$
\begin{aligned}
g_{n} g_{n+1} & =\left\{f_{n-1} f_{n}-\frac{1}{3}(-1)^{n}\right\}\left\{f_{n} f_{n+1}+\frac{1}{3}(-1)^{n}\right\} \\
& =f_{n}^{2}\left(f_{n+1} f_{n-1}\right)-\frac{1}{3}(-1)^{n} f_{n}\left(f_{n+1}-f_{n-1}\right)-\frac{1}{9} \\
& =f_{n}^{2}\left(f_{n}^{2}+(-1)^{n}\right)-\frac{1}{3}(-1)^{n} f_{n}^{2}-\frac{1}{9} \\
& =f_{n}^{4}+\frac{2}{3}(-1)^{n} f_{n}^{2}-\frac{1}{9} .
\end{aligned}
$$

The desired result comes from the above two identities.

Now we prove Theorem 2.1. By Proposition 2.2, we have

$$
\frac{1}{g_{n}}-\frac{1}{g_{n+1}}-\frac{1}{f_{n}^{2}}=\frac{\left(g_{n+1}-g_{n}\right) f_{n}^{2}-g_{n} g_{n+1}}{g_{n} g_{n+1} f_{n}^{2}}=\frac{1}{9 g_{n} g_{n+1} f_{n}^{2}}>0 .
$$

It follows that

$$
\frac{1}{g_{n}}>\frac{1}{f_{n}^{2}}+\frac{1}{g_{n+1}}
$$

for all $n \in \mathbb{N}$. By Lemma 1.1, we obtain

$$
\frac{1}{g_{n}}>\sum_{k=n}^{\infty} \frac{1}{f_{k}^{2}} .
$$

For the proof of the converse inequality, we compute

$$
\begin{aligned}
\frac{1}{g_{n}+c_{n}}-\frac{1}{g_{n+1}+c_{n+1}}-\frac{1}{f_{n}^{2}} & =\frac{\left(g_{n+1}-g_{n}\right)-\left(c_{n}-c_{n+1}\right)}{\left(g_{n}+c_{n}\right)\left(g_{n+1}+c_{n+1}\right)}-\frac{1}{f_{n}^{2}} \\
& <\frac{g_{n+1}-g_{n}}{\left(g_{n}+c_{n}\right)\left(g_{n+1}+c_{n+1}\right)}-\frac{1}{f_{n}^{2}} \\
& =\frac{A}{\left(g_{n}+c_{n}\right)\left(g_{n+1}+c_{n+1}\right) f_{n}^{2}},
\end{aligned}
$$

where

$$
A=\left(g_{n+1}-g_{n}\right) f_{n}^{2}-\left(g_{n}+c_{n}\right)\left(g_{n+1}+c_{n+1}\right) .
$$

By Proposition 2.2, we have

$$
A=\frac{1}{9}-c_{n} g_{n+1}-c_{n+1} g_{n}-c_{n} c_{n+1}<\frac{1}{9}-c_{n} g_{n+1}<0,
$$


Table 1 Some values of $\left(\sum_{k=n}^{\infty} \frac{1}{f_{k}^{2}}\right)^{-1}$

\begin{tabular}{rrrrrr}
\hline$n$ & $\left(\sum_{k=n}^{\infty} \frac{1}{f_{k}^{2}}\right)^{-1}$ & $f_{n}^{2}-f_{n-1}^{2}$ & $n$ & $\left(\sum_{k=n}^{\infty} \frac{1}{f_{k}^{2}}\right)^{-1}$ & $f_{n}^{2}-f_{n-1}^{2}$ \\
\hline 3 & $2.3456 \ldots$ & 3 & 4 & $5.6714 \ldots$ & 5 \\
5 & $15.3351 \ldots$ & 16 & 6 & $39.6673 \ldots$ & 39 \\
7 & $104.3335 \ldots$ & 105 & 8 & $272.6667 \ldots$ & 272 \\
9 & $714.3333 \ldots$ & 715 & 10 & $1869.6666 \ldots$ & 1869 \\
\hline
\end{tabular}

since $c_{n} g_{n+1}=f_{n+1}-\frac{\frac{1}{3}(-1)^{n+1}}{f_{n}} \geq f_{n+1}-\frac{1}{3} \geq \frac{2}{3}$ for all $n \in \mathbb{N}$. Thus we obtain

$$
\frac{1}{g_{n}+c_{n}}<\frac{1}{f_{n}^{2}}+\frac{1}{g_{n+1}+c_{n+1}}
$$

for all $n \in \mathbb{N}$. By Lemma 1.1 again, we obtain

$$
\frac{1}{g_{n}+c_{n}}<\sum_{k=n}^{\infty} \frac{1}{f_{k}^{2}}
$$

By (2.2) and (2.3), we complete the proof of Theorem 2.1.

Remark 2.3 The inequalities of Theorem 2.1 imply

$$
\lim _{n \rightarrow \infty}\left\{\left(\sum_{k=n}^{\infty} \frac{1}{f_{k}^{2}}\right)^{-1}-g_{n}\right\}=0
$$

See Table 1.

\section{Reciprocal sum of $f_{k} f_{k+m}$ when $m$ is even}

In Sects. 3 and 4, we deal with the value of

$$
\left(\sum_{k=n}^{\infty} \frac{1}{f_{k} f_{k+m}}\right)^{-1}
$$

In fact, we can compute the explicit value when $m=2$. Note that

$$
\begin{aligned}
\sum_{k=n}^{\infty} \frac{1}{f_{k} f_{k+2}} & =\sum_{k=n}^{\infty}\left(\frac{1}{f_{k}}-\frac{1}{f_{k+2}}\right) \frac{1}{f_{k+2}-f_{k}} \\
& =\sum_{k=n}^{\infty}\left(\frac{1}{f_{k} f_{k+1}}-\frac{1}{f_{k+1} f_{k+2}}\right) \\
& =\frac{1}{f_{n} f_{n+1}} .
\end{aligned}
$$

Hence it holds that, for all $n \in \mathbb{N}$,

$$
\left(\sum_{k=n}^{\infty} \frac{1}{f_{k} f_{k+2}}\right)^{-1}=f_{n} f_{n+1} .
$$


However, it is difficult to find the explicit value of

$$
\left(\sum_{k=n}^{\infty} \frac{1}{f_{k} f_{k+m}}\right)^{-1}
$$

except for $m=2$.

Throughout this section, we assume that $m$ is even, so that $m=2 \ell$ for some $\ell \in \mathbb{N}$. In this case, we define

$$
g_{n}=g_{n, \ell}=f_{n+\ell-1} f_{n+\ell}-\left(f_{\ell}^{2}+(-1)^{\ell}\right) \frac{(-1)^{n}}{3} .
$$

For simplicity, we write $I_{1}:=f_{\ell}^{2}+(-1)^{\ell}$.

Proposition 3.1 For any $n \in \mathbb{N}$, we have

$$
\left(g_{n+1}-g_{n}\right) f_{n} f_{n+2 \ell}-g_{n} g_{n+1}=-\frac{5}{9} I_{1}^{2}+\frac{2}{3}(-1)^{\ell} I_{1} .
$$

Proof Write $g_{n}=f_{n+\ell-1} f_{n+\ell}-\frac{(-1)^{n}}{3} I_{1}$. Note that

$$
\begin{aligned}
& \left(g_{n+1}-g_{n}\right) f_{n} f_{n+2 \ell} \\
& \quad=\left\{\left(f_{n+\ell} f_{n+\ell+1}+\frac{(-1)^{n}}{3} I_{1}\right)-\left(f_{n+\ell-1} f_{n+\ell}-\frac{(-1)^{n}}{3} I_{1}\right)\right\} f_{n} f_{n+2 \ell} \\
& \quad=\left\{f_{n+\ell}^{2}+\frac{2}{3}(-1)^{n} I_{1}\right\}\left\{f_{n+\ell}^{2}-(-1)^{n} f_{\ell}^{2}\right\} .
\end{aligned}
$$

Since $f_{\ell}^{2}=I_{1}-(-1)^{\ell}$, we have

$$
\begin{aligned}
& \left(g_{n+1}-g_{n}\right) f_{n} f_{n+2 \ell} \\
& \quad=f_{n+\ell}^{4}+(-1)^{n+\ell} f_{n+\ell}^{2}-\frac{(-1)^{n}}{3} I_{1} f_{n+\ell}^{2}-\frac{2}{3} I_{1}^{2}+\frac{2}{3}(-1)^{\ell} I_{1} .
\end{aligned}
$$

Note that

$$
\begin{aligned}
g_{n} g_{n+1} & =\left(f_{n+\ell-1} f_{n+\ell}-\frac{(-1)^{n}}{3} I_{1}\right)\left(f_{n+\ell} f_{n+\ell+1}+\frac{(-1)^{n}}{3} I_{1}\right) \\
& =f_{n+\ell}^{2} f_{n+\ell-1} f_{n+\ell+1}-\frac{(-1)^{n}}{3} I_{1} f_{n+\ell}^{2}-\frac{1}{9} I_{1}^{2} .
\end{aligned}
$$

By Lemma 1.2, $f_{n+\ell-1} f_{n+\ell+1}=f_{n+\ell}^{2}+(-1)^{n+\ell}$. Thus we have

$$
g_{n} g_{n+1}=f_{n+\ell}^{4}+(-1)^{n+\ell} f_{n+\ell}^{2}-\frac{(-1)^{n}}{3} I_{1} f_{n+\ell}^{2}-\frac{1}{9} I_{1}^{2} .
$$

By (3.1) and (3.2), we complete the proof.

See Table 2. If $m=4$, then $g_{n, 2}=f_{n+1} f_{n+2}-\frac{2}{3}(-1)^{n}$. 
Table 2 Some values of $\left(\sum_{k=n}^{\infty} \frac{1}{f_{k} f_{k+4}}\right)^{-1}$

\begin{tabular}{rrrrrr}
\hline$n$ & $\left(\sum_{k=n}^{\infty} \frac{1}{f_{k} f_{k+4}}\right)^{-1}$ & \multicolumn{1}{c}{$g_{n, 2}$} & $n$ & \multicolumn{1}{c}{$\left(\sum_{k=n}^{\infty} \frac{1}{f_{k} f_{k+4}}\right)^{-1}$} & \multicolumn{1}{c}{$g_{n, 2}$} \\
\hline 3 & $15.6521 \ldots$ & 15.6666 & 4 & $39.3277 \ldots$ & 39.3333 \\
5 & $104.6645 \ldots$ & 104.6666 & 6 & $272.3325 \ldots$ & 272.3333 \\
7 & $714.6663 \ldots$ & 714.6666 & 8 & $1869.3332 \ldots$ & 1869.3333 \\
9 & $4895.6666 \ldots$ & 4895.6666 & 10 & $12,815.3333 \ldots$ & $12,815.3333$ \\
\hline
\end{tabular}

Theorem 3.2 If $m=2 \ell$ for some $\ell \in \mathbb{N}$, then for any $n \geq 2 \ell-1$, we have

$$
g_{n}-c_{n}<\left(\sum_{k=n}^{\infty} \frac{1}{f_{k} f_{k+m}}\right)^{-1} \leq g_{n}
$$

where $c_{n}=1 / f_{n}$.

For example, if $\ell=4$, then $g_{n}=f_{n+3} f_{n+4}-\frac{10(-1)^{n}}{3}$. Thus we have

$$
\left\lfloor\left(\sum_{k=n}^{\infty} \frac{1}{f_{k} f_{k+8}}\right)^{-1}\right\rfloor= \begin{cases}f_{n+3} f_{n+4}-4, & n \text { is even; } \\ f_{n+3} f_{n+4}+3, & n \text { is odd }\end{cases}
$$

Proof (i) By Lemma 1.1, it is enough to show that

$$
\frac{1}{g_{n}} \leq \frac{1}{f_{n} f_{n+2 \ell}}+\frac{1}{g_{n+1}}
$$

for all $n \in \mathbb{N}$. By Proposition 3.1, we have

$$
\begin{aligned}
\left(g_{n+1}-g_{n}\right) f_{n} f_{n+2 \ell}-g_{n} g_{n+1} & =-\frac{5}{9} I_{1}^{2}+\frac{2}{3}(-1)^{\ell} I_{1} \\
& =-\frac{I_{1}}{9}\left(5 I_{1}-6(-1)^{\ell}\right) \\
& =-\frac{I_{1}}{9}\left(5 f_{\ell}^{2}-(-1)^{\ell}\right) \leq 0 .
\end{aligned}
$$

It follows that

$$
\frac{1}{g_{n}}-\frac{1}{g_{n+1}}-\frac{1}{f_{n} f_{n+2 \ell}}=\frac{\left(g_{n+1}-g_{n}\right) f_{n} f_{n+2 \ell}-g_{n} g_{n+1}}{g_{n} g_{n+2} f_{n} f_{n+2 \ell}} \leq 0,
$$

which implies (3.3). In fact, the equality holds only when $\ell=1$. This is the case when $m=2$.

(ii) By Lemma 1.1, it is enough to show that

$$
\frac{1}{g_{n}-c_{n}}>\frac{1}{f_{n} f_{n+2 \ell}}+\frac{1}{g_{n+1}-c_{n+1}}
$$

for all $n \in \mathbb{N}$. Note that

$$
\begin{aligned}
& \frac{1}{g_{n}-c_{n}}-\frac{1}{g_{n+1}-c_{n+1}}-\frac{1}{f_{n} f_{n+2 \ell}} \\
& \quad=\frac{\left(g_{n+1}-g_{n}\right)+\left(c_{n}-c_{n+1}\right)}{\left(g_{n}-c_{n}\right)\left(g_{n+1}-c_{n+1}\right)}-\frac{1}{f_{n} f_{n+2 \ell}}
\end{aligned}
$$




$$
\begin{aligned}
& >\frac{g_{n+1}-g_{n}}{\left(g_{n}-c_{n}\right)\left(g_{n+1}-c_{n+1}\right)}-\frac{1}{f_{n} f_{n+2 \ell}} \\
& =\frac{\left(g_{n+1}-g_{n}\right) f_{n} f_{n+2 \ell}-\left(g_{n}-c_{n}\right)\left(g_{n+1}-c_{n+1}\right)}{\left(g_{n}-c_{n}\right)\left(g_{n+1}-c_{n+1}\right) f_{n} f_{n+2 \ell}} .
\end{aligned}
$$

Now we will show that, for $n \geq 2 \ell-1$, we have

$$
\left(g_{n+1}-g_{n}\right) f_{n} f_{n+2 \ell}-\left(g_{n}-c_{n}\right)\left(g_{n+1}-c_{n+1}\right)>0 .
$$

Note that

$$
\begin{aligned}
& \left(g_{n+1}-g_{n}\right) f_{n} f_{n+2 \ell}-\left(g_{n}-c_{n}\right)\left(g_{n+1}-c_{n+1}\right) \\
& \quad=\left\{\left(g_{n+1}-g_{n}\right) f_{n} f_{n+2 \ell}-g_{n} g_{n+1}\right\}+\left\{c_{n} g_{n+1}+c_{n+1} g_{n}-c_{n} c_{n+1}\right\} .
\end{aligned}
$$

By Proposition 3.1, we have

$$
\begin{aligned}
\left(g_{n+1}-g_{n}\right) f_{n} f_{n+2 \ell}-g_{n} g_{n+1} & =-\frac{5}{9} I_{1}^{2}+\frac{2}{3}(-1)^{\ell} I_{1} \\
& =-\frac{5}{9} f_{\ell}^{4}-\frac{4}{9}(-1)^{\ell} f_{\ell}^{2}+\frac{1}{9} \\
& >-\frac{5}{9} f_{\ell}^{4}-\frac{4}{9} f_{\ell}^{2}+\frac{1}{9} .
\end{aligned}
$$

By Lemma 1.3, we have

$$
\begin{aligned}
& c_{n} g_{n+1}+c_{n+1} g_{n}-c_{n} c_{n+1} \\
& \quad=\frac{f_{n+\ell} f_{n+\ell+1}}{f_{n}}+\frac{f_{n+\ell-1} f_{n+\ell}}{f_{n+1}}+\frac{(-1)^{n}}{3}\left(\frac{1}{f_{n}}-\frac{1}{f_{n+1}}\right) I_{1}-\frac{1}{f_{n} f_{n+1}} \\
& \quad>f_{\ell+1} f_{n+\ell+1}+f_{\ell} f_{n+\ell-1}-\frac{1}{3} I_{1}-1 \\
& \quad>f_{\ell}\left(f_{n+\ell+1}+f_{n+\ell-1}\right)-\frac{1}{3} I_{1}-1 \\
& \quad>f_{\ell}^{4}\left(f_{n-2 \ell+4}+f_{n-2 \ell+2}\right)-\frac{1}{3} I_{1}-1 .
\end{aligned}
$$

If $n \geq 2 \ell-1$, then $f_{n-2 \ell+4}+f_{n-2 \ell+2} \geq f_{3}+f_{1}=3$. Thus, we have

$$
c_{n} g_{n+1}+c_{n+1} g_{n}-c_{n} c_{n+1}>3 f_{\ell}^{4}-\frac{1}{3} I_{1}-1>3 f_{\ell}^{4}-\frac{1}{3} f_{\ell}^{2}-\frac{4}{3}
$$

for $n \geq 2 \ell-1$. From (3.4) and (3.5), we obtain

$$
\left(g_{n+1}-g_{n}\right) f_{n} f_{n+2 \ell}-\left(g_{n}-c_{n}\right)\left(g_{n+1}-c_{n+1}\right)>\frac{22}{9} f_{\ell}^{4}-\frac{7}{9} f_{\ell}^{2}-\frac{11}{9} \geq \frac{4}{9}>0,
$$

since $f_{\ell} \geq 1$.

Remark 3.3 If $\ell=0$, then $I_{1}=f_{0}^{2}+1=1$. Thus in the proof of Theorem 3.2 we have

$$
\left(g_{n+1}-g_{n}\right) f_{n} f_{n+2 \ell}-g_{n} g_{n+1}=-\frac{I_{1}}{9}\left(5 f_{\ell}^{2}-(-1)^{\ell}\right)=\frac{1}{9}>0 .
$$


In fact, this is an identity in Proposition 2.2. Thus the inequality in Theorem 3.2 is opposite to Theorem 2.1.

\section{Reciprocal sum of $f_{k} f_{k+m}$ when $m$ is odd}

Throughout this section, we assume that $m$ is odd, so that $m=2 \ell-1$ for some $\ell \in \mathbb{N}$. In this case, we define

$$
h_{n}=h_{n, \ell}=f_{n+\ell-1}^{2}-\left(f_{\ell-1} f_{\ell}+(-1)^{\ell}\right) \frac{(-1)^{n}}{3} .
$$

For simplicity, we write $I_{2}:=f_{\ell-1} f_{\ell}+(-1)^{\ell}$.

Proposition 4.1 For any $n \in \mathbb{N}$, we have

$$
\left(h_{n+1}-h_{n}\right) f_{n} f_{n+2 \ell-1}-h_{n} h_{n+1}=-1+\frac{4}{3}(-1)^{\ell} I_{2}-\frac{5}{9} I_{2}^{2} .
$$

Proof By Catalan's identity, we have

$$
\begin{aligned}
f_{n} f_{n+2 \ell-1} & =f_{n}\left(f_{n+2 \ell}-f_{n+2 \ell-2}\right) \\
& =f_{n} f_{n+2 \ell}-f_{n} f_{n+2 \ell-2} \\
& =\left(f_{n+\ell}^{2}-(-1)^{n} f_{\ell}^{2}\right)-\left(f_{n+\ell-1}^{2}-(-1)^{n} f_{\ell-1}^{2}\right) \\
& =f_{n+\ell+1} f_{n+\ell-2}-(-1)^{n} f_{\ell+1} f_{\ell-2} .
\end{aligned}
$$

Note that

$$
\begin{aligned}
\left(h_{n+1}-h_{n}\right) f_{n} f_{n+2 \ell-1} & \\
& =\left\{\left(f_{n+\ell}^{2}-f_{n+\ell-1}^{2}\right)+\frac{2}{3}(-1)^{n} I_{2}\right\} f_{n} f_{n+2 \ell-1} \\
& =\left\{f_{n+\ell+1} f_{n+\ell-2}+\frac{2}{3}(-1)^{n} I_{2}\right\}\left\{f_{n+\ell+1} f_{n+\ell-2}-(-1)^{n} f_{\ell+1} f_{\ell-2}\right\} \\
& =\left(f_{n+\ell+1} f_{n+\ell-2}\right)^{2}+f_{n+\ell+1} f_{n+\ell-2}(-1)^{n}\left\{\frac{2}{3} I_{2}-f_{\ell+1} f_{\ell-2}\right\}-\frac{2}{3} f_{\ell+1} f_{\ell-2} I_{2} .
\end{aligned}
$$

Note that

$$
\begin{aligned}
h_{n} h_{n+1} & =\left\{f_{n+\ell-1}^{2}-\frac{(-1)^{n}}{3} I_{2}\right\}\left\{f_{n+\ell}^{2}+\frac{(-1)^{n}}{3} I_{2}\right\} \\
& =\left(f_{n+\ell-1} f_{n+\ell}\right)^{2}-\left(f_{n+\ell}^{2}-f_{n+\ell-1}^{2}\right) \frac{(-1)^{n}}{3} I_{2}-\frac{1}{9} I_{2}^{2} \\
& =\left(f_{n+\ell-1} f_{n+\ell}\right)^{2}-f_{n+\ell+1} f_{n+\ell-2} \frac{(-1)^{n}}{3} I_{2}-\frac{1}{9} I_{2}^{2} .
\end{aligned}
$$

It follows that

$$
\begin{aligned}
\left(h_{n+1}-h_{n}\right) f_{n} f_{n+2 \ell-1}-h_{n} h_{n+1}= & \left(f_{n+\ell+1} f_{n+\ell-2}\right)^{2}-\left(f_{n+\ell-1} f_{n+\ell}\right)^{2} \\
& +f_{n+\ell+1} f_{n+\ell-2}(-1)^{n}\left\{I_{2}-f_{\ell+1} f_{\ell-2}\right\} \\
& -\frac{2}{3} f_{\ell+1} f_{\ell-2} I_{2}+\frac{1}{9} I_{2}^{2} .
\end{aligned}
$$


Table 3 Some values of $\left(\sum_{k=n}^{\infty} \frac{1}{f_{k} f_{k+5}}\right)^{-1}$

\begin{tabular}{rrrrrr}
\hline$n$ & $\left(\sum_{k=n}^{\infty} \frac{1}{f_{k} f_{k+5}}\right)^{-1}$ & \multicolumn{1}{c}{$h_{n, 3}$} & $n$ & \multicolumn{1}{c}{$\left(\sum_{k=n}^{\infty} \frac{1}{f_{k} f_{k+5}}\right)^{-1}$} & \multicolumn{1}{c}{$h_{n, 3}$} \\
\hline 3 & $25.3042 \ldots$ & $25.3333 \ldots$ & 4 & $63.6554 \ldots$ & $63.6666 \ldots$ \\
5 & $169.3290 \ldots$ & $169.3333 \ldots$ & 6 & $440.6650 \ldots$ & $440.6666 \ldots$ \\
7 & $1156.3327 \ldots$ & $1156.3333 \ldots$ & 8 & $3024.6664 \ldots$ & $3024.6666 \ldots$ \\
9 & $7921.3332 \ldots$ & $7921.3333 \ldots$ & 10 & $20,735.6666 \ldots$ & $20,735.6666 \ldots$ \\
\hline
\end{tabular}

Since $f_{n+\ell-1} f_{n+\ell}=f_{n+\ell+1} f_{n+\ell-2}+(-1)^{n+\ell}$, we have

$$
\begin{aligned}
& \left(f_{n+\ell+1} f_{n+\ell-2}\right)^{2}-\left(f_{n+\ell-1} f_{n+\ell}\right)^{2} \\
& \quad=\left(f_{n+\ell+1} f_{n+\ell-2}+f_{n+\ell-1} f_{n+\ell}\right)\left(f_{n+\ell+1} f_{n+\ell-2}-f_{n+\ell-1} f_{n+\ell}\right) \\
& \quad=-1-2(-1)^{n+\ell} f_{n+\ell+1} f_{n+\ell-2} .
\end{aligned}
$$

Since $I_{2}-f_{\ell+1} f_{\ell-2}=f_{\ell-1} f_{\ell}-f_{\ell+1} f_{\ell-2}+(-1)^{\ell}=2(-1)^{\ell}$,

$$
\begin{aligned}
\left(h_{n+1}\right. & \left.-h_{n}\right) f_{n} f_{n+2 \ell-1}-h_{n} h_{n+1} \\
= & -1-2(-1)^{n+\ell} f_{n+\ell+1} f_{n+\ell-2} \\
& \quad+f_{n+\ell+1} f_{n+\ell-2}(-1)^{n}\left\{2(-1)^{\ell}\right\}-\frac{2}{3} f_{\ell+1} f_{\ell-2} I_{2}+\frac{1}{9} I_{2}^{2} \\
= & -1-\frac{2}{3}\left(I_{2}-2(-1)^{\ell}\right) I_{2}+\frac{1}{9} I_{2}^{2} \\
= & -1+\frac{4}{3}(-1)^{\ell} I_{2}-\frac{5}{9} I_{2}^{2} .
\end{aligned}
$$

See Table 3. If $m=5$, then $h_{n, 3}=f_{n+2}^{2}-\frac{1}{3}(-1)^{n}$.

Theorem 4.2 If $m=2 \ell-1$, then for any $n \geq 2 \ell-2$, we have

$$
h_{n}-c_{n}<\left(\sum_{k=n}^{\infty} \frac{1}{f_{k} f_{k+m}}\right)^{-1}<h_{n}
$$

where $c_{n}=1 / f_{n}$.

For example, if $\ell=4$, then $h_{n}=f_{n+3}^{2}-\frac{7(-1)^{n}}{3}$. Thus we have

$$
\left\lfloor\left(\sum_{k=n}^{\infty} \frac{1}{f_{k} f_{k+7}}\right)^{-1}\right\rfloor= \begin{cases}f_{n+3}^{2}-3, & n \text { is even; } \\ f_{n+3}^{2}+2, & n \text { is odd }\end{cases}
$$

Proof (i) We will show that

$$
\frac{1}{h_{n}}<\frac{1}{f_{n} f_{n+2 \ell-1}}+\frac{1}{h_{n+1}} .
$$

Note that

$$
\frac{1}{h_{n}}-\frac{1}{h_{n+1}}-\frac{1}{f_{n} f_{n+2 \ell-1}}=\frac{\left(h_{n+1}-h_{n}\right) f_{n} f_{n+2 \ell-1}-h_{n} h_{n+1}}{h_{n} h_{n+1} f_{n} f_{n+2 \ell-1}} .
$$


By Proposition 4.1, we have

$$
\begin{aligned}
\left(h_{n+1}-h_{n}\right) f_{n} f_{n+2 \ell-1}-h_{n} h_{n+1} & =-1+\frac{4}{3}(-1)^{\ell} I_{2}-\frac{5}{9} I_{2}^{2} \\
& =-\frac{5}{9}\left(f_{\ell-1} f_{\ell}\right)^{2}+\frac{2}{9}(-1)^{\ell}\left(f_{\ell-1} f_{\ell}\right)-\frac{2}{9} \\
& \leq-\frac{5}{9}\left(f_{\ell-1} f_{\ell}\right)^{2}+\frac{2}{9}\left(f_{\ell-1} f_{\ell}\right)-\frac{2}{9} \\
& <-\frac{5}{9}<0 .
\end{aligned}
$$

(ii) We will show that

$$
\frac{1}{h_{n}-c_{n}}>\frac{1}{f_{n} f_{n+2 \ell-1}}+\frac{1}{h_{n+1}-c_{n+1}} .
$$

Note that

$$
\begin{aligned}
\frac{1}{h_{n}-c_{n}}-\frac{1}{h_{n+1}-c_{n+1}}-\frac{1}{f_{n} f_{n+2 \ell-1}} \\
=\frac{h_{n+1}-h_{n}+\left(c_{n}-c_{n+1}\right)}{\left(h_{n}-c_{n}\right)\left(h_{n+1}-c_{n+1}\right)}-\frac{1}{f_{n} f_{n+2 \ell-1}} \\
>\frac{h_{n+1}-h_{n}}{\left(h_{n}-c_{n}\right)\left(h_{n+1}-c_{n+1}\right)}-\frac{1}{f_{n} f_{n+2 \ell-1}} \\
=\frac{\left(h_{n+1}-h_{n}\right) f_{n} f_{n+2 \ell-1}-\left(h_{n}-c_{n}\right)\left(h_{n+1}-c_{n+1}\right)}{\left(h_{n}-c_{n}\right)\left(h_{n+1}-c_{n+1}\right) f_{n} f_{n+2 \ell-1}} .
\end{aligned}
$$

It is enough to show that

$$
\left(h_{n+1}-h_{n}\right) f_{n} f_{n+2 \ell-1}-\left(h_{n}-c_{n}\right)\left(h_{n+1}-c_{n+1}\right)>0 .
$$

Note that

$$
\begin{aligned}
& \left(h_{n+1}-h_{n}\right) f_{n} f_{n+2 \ell-1}-\left(h_{n}-c_{n}\right)\left(h_{n+1}-c_{n+1}\right) \\
& \quad=\left\{\left(h_{n+1}-h_{n}\right) f_{n} f_{n+2 \ell-1}-h_{n} h_{n+1}\right\}+\left\{c_{n} h_{n+1}+c_{n+1} h_{n}-c_{n} c_{n+1}\right\} .
\end{aligned}
$$

By Proposition 4.1, we have

$$
\begin{aligned}
\left(h_{n+1}-h_{n}\right) f_{n} f_{n+2 \ell-1}-h_{n} h_{n+1} & =-1+\frac{4}{3}(-1)^{\ell} I_{2}-\frac{5}{9} I_{2}^{2} \\
& =-\frac{5}{9}\left(f_{\ell-1} f_{\ell}\right)^{2}+\frac{2}{9}(-1)^{\ell}\left(f_{\ell-1} f_{\ell}\right)-\frac{2}{9} \\
& \geq-\frac{5}{9}\left(f_{\ell-1} f_{\ell}\right)^{2}-\frac{2}{9}\left(f_{\ell-1} f_{\ell}\right)-\frac{2}{9} .
\end{aligned}
$$

Note that

$$
\begin{aligned}
c_{n} h_{n+1}+c_{n+1} h_{n}-c_{n} c_{n+1} \\
\quad=\frac{f_{n+\ell}^{2}}{f_{n}}+\frac{f_{n+\ell-1}^{2}}{f_{n+1}}+\frac{(-1)^{n}}{3} I_{2}\left(\frac{1}{f_{n}}-\frac{1}{f_{n+1}}\right)-\frac{1}{f_{n} f_{n+1}}
\end{aligned}
$$




$$
\begin{aligned}
& >f_{\ell+1} f_{n+\ell}+f_{\ell-1} f_{n+\ell-1}-\frac{1}{3} I_{2}-1 \\
& >f_{\ell-1}\left(f_{n+\ell}+f_{n+\ell-1}\right)-\frac{1}{3} I_{2}-1 \\
& >\left(f_{\ell-1} f_{\ell}\right)^{2}\left(f_{n-2 \ell+5}+f_{n-2 \ell+4}\right)-\frac{1}{3} I_{2}-1 .
\end{aligned}
$$

If $n \geq 2 \ell-2$, then $f_{n-2 \ell+5}+f_{n-2 \ell+4} \geq f_{3}+f_{2}=3$. It follows that

$$
c_{n} h_{n+1}+c_{n+1} h_{n}-c_{n} c_{n+1}>3\left(f_{\ell-1} f_{\ell}\right)^{2}-\frac{1}{3}\left(f_{\ell-1} f_{\ell}\right)-\frac{4}{3} .
$$

Thus we have

$$
\begin{gathered}
\left(h_{n+1}-h_{n}\right) f_{n} f_{n+2 \ell-1}-\left(h_{n}-c_{n}\right)\left(h_{n+1}-c_{n+1}\right) \\
>\frac{22}{9}\left(f_{\ell-1} f_{\ell}\right)^{2}-\frac{5}{9}\left(f_{\ell-1} f_{\ell}\right)-\frac{14}{9} \geq \frac{1}{3}>0 .
\end{gathered}
$$

\section{Reciprocal sum of $f_{3 k}^{2}$}

In this final section, we discuss the reciprocal sum of $f_{m k}^{2}$ for any $m \geq 2$. Similar to (2.1), when $m=1$, we expected to find a suitable constant $C_{m}>0$ such that

$$
\left(\sum_{k=n}^{\infty} \frac{1}{f_{m k}^{2}}\right)^{-1} \sim f_{m n}^{2}-f_{m(n-1)}^{2}+(-1)^{m n} C_{m} .
$$

In Sect. 2, we proved that $C_{1}=\frac{2}{3}$. The integer part when $m=3$ has been obtained as follows.

Theorem $5.1([5])$

$$
\left\lfloor\left(\sum_{k=n}^{\infty} \frac{1}{f_{3 k}^{2}}\right)^{-1}\right\rfloor= \begin{cases}f_{3 n}^{2}-f_{3 n-3}^{2}, & n \text { is even } \\ f_{3 n}^{2}-f_{3 n-3}^{2}-1, & n \text { is odd }\end{cases}
$$

\begin{tabular}{|c|c|c|c|c|c|}
\hline$n$ & $\left(\sum_{k=n}^{\infty} \frac{1}{f_{3 k}^{2}}\right)^{-1}$ & $\tilde{g}_{n}$ & $n$ & $\left(\sum_{k=n}^{\infty} \frac{1}{f_{3 k}^{2}}\right)^{-1}$ & $\tilde{g}_{n}$ \\
\hline 3 & $1091.5561 \ldots$ & $1091.5555 \ldots$ & 4 & $19,580.44447 \ldots$ & $19,580.44444 \ldots$ \\
\hline 5 & $351,363.555557 \ldots$ & $351,363.555555 \ldots$ & 6 & $6,304,956.4444445 \ldots$ & $6,304,956.4444444 \ldots$ \\
\hline 7 & $113,137,859.555555 \ldots$ & $113,137,859.555555 \ldots$ & & & \\
\hline
\end{tabular}

Now we prove that $C_{3}=\frac{4}{9}$. See Table 4 . Here

$$
\widetilde{g}_{n}:=f_{3 n}^{2}-f_{3 n-3}^{2}+\frac{4}{9}(-1)^{n} .
$$

Our theorem contains more optimal inequality than (5.2).

Theorem 5.2 For all $n \in \mathbb{N}$, we have

$$
\widetilde{g}_{n}<\left(\sum_{k=n}^{\infty} \frac{1}{f_{3 k}^{2}}\right)^{-1}<\widetilde{g}_{n}+c_{n},
$$

Table 4 Some values of $\left(\sum_{k=n}^{\infty} \frac{1}{f_{3 k}^{2}}\right)^{-1}$ 
where $\widetilde{g}_{n}:=f_{3 n}^{2}-f_{3 n-3}^{2}+\frac{4}{9}(-1)^{n}$. Thus we have

$$
\left(\sum_{k=n}^{\infty} \frac{1}{f_{3 k}^{2}}\right)^{-1} \sim f_{3 n}^{2}-f_{3 n-3}^{2}+\frac{4}{9}(-1)^{n} .
$$

Lemma 5.3 For any $n \geq 1$, we have

$$
f_{3 n+3}^{2}-18 f_{3 n}^{2}+f_{3 n-3}^{2}=8(-1)^{n}
$$

Proof Since $f_{3 n+2}+f_{3 n-2}=3 f_{3 n}$, we have

$$
\begin{aligned}
f_{3 n+3} & =f_{3 n+2}+f_{3 n}+f_{3 n-1} \\
& =f_{3 n+2}+f_{3 n}+f_{3 n-2}+f_{3 n-3} \\
& =4 f_{3 n}+f_{3 n-3} .
\end{aligned}
$$

It follows that

$$
\begin{aligned}
16 f_{3 n}^{2} & =\left(f_{3 n+3}-f_{3 n-3}\right)^{2} \\
& =f_{3 n+3}^{2}+f_{3 n-3}^{2}-2 f_{3 n+3} f_{3 n-3} \\
& =f_{3 n+3}^{2}+f_{3 n-3}^{2}-2\left\{f_{3 n}^{2}+(-1)^{n} f_{3}^{2}\right\},
\end{aligned}
$$

which completes the proof.

Proof (i) We will show that

$$
\frac{1}{\widetilde{g}_{n}}>\frac{1}{f_{3 n}^{2}}+\frac{1}{\widetilde{g}_{n+1}} .
$$

Note that

$$
\frac{1}{\widetilde{g}_{n}}-\frac{1}{\widetilde{g}_{n+1}}-\frac{1}{f_{3 n}^{2}}=\frac{A}{\widetilde{g}_{n} \widetilde{g}_{n+1} f_{3 n}^{2}},
$$

where $A:=\left(\widetilde{g}_{n+1}-\widetilde{g}_{n}\right) f_{3 n}^{2}-\widetilde{g}_{n} \widetilde{g}_{n+1}$. It is enough to show that $A>0$.

Note that

$$
\left(\widetilde{g}_{n+1}-\widetilde{g}_{n}\right) f_{3 n}^{2}=f_{3 n+3}^{2} f_{3 n}^{2}-2 f_{3 n}^{4}+f_{3 n-3}^{2} f_{3 n}^{2}-\frac{8}{9}(-1)^{n} f_{3 n}^{2} .
$$

Note also that

$$
\begin{aligned}
\tilde{g}_{n} \widetilde{g}_{n+1}= & \left(f_{3 n}^{2}-f_{3 n-3}^{2}+\frac{4}{9}(-1)^{n}\right)\left(f_{3 n+3}^{2}-f_{3 n}^{2}-\frac{4}{9}(-1)^{n}\right) \\
= & f_{3 n}^{2} f_{3 n+3}^{2}-f_{3 n}^{4}-f_{3 n-3}^{2} f_{3 n+3}^{2}+f_{3 n-3}^{2} f_{3 n}^{2}-\frac{16}{81} \\
& +\frac{4}{9}(-1)^{n}\left(f_{3 n+3}^{2}-2 f_{3 n}^{2}+f_{3 n-3}^{2}\right) .
\end{aligned}
$$


It follows that

$$
\begin{aligned}
A & =f_{3 n-3}^{2} f_{3 n+3}^{2}-f_{3 n}^{4}-\frac{4}{9}(-1)^{n}\left\{f_{3 n+3}^{2}+f_{3 n-3}^{2}\right\}+\frac{16}{81} \\
& =\left(f_{3 n}^{2}+(-1)^{n} f_{3}^{2}\right)^{2}-f_{3 n}^{4}-\frac{4}{9}(-1)^{n}\left\{f_{3 n+3}^{2}+f_{3 n-3}^{2}\right\}+\frac{16}{81} \\
& =-\frac{4}{9}(-1)^{n}\left\{f_{3 n+3}^{2}-18 f_{3 n}^{2}+f_{3 n-3}^{2}\right\}+\frac{1312}{81} .
\end{aligned}
$$

By Lemma 5.3, we have

$$
A=\frac{1024}{81}
$$

(ii) We will prove that

$$
\frac{1}{\widetilde{g}_{n}+c_{n}}<\frac{1}{f_{3 n}^{2}}+\frac{1}{\widetilde{g}_{n+1}+c_{n+1}} .
$$

Note that

$$
\begin{aligned}
\frac{1}{\widetilde{g}_{n}+c_{n}}-\frac{1}{\widetilde{g}_{n+1}+c_{n+1}}-\frac{1}{f_{3 n}^{2}} & =\frac{\left(\widetilde{g}_{n+1}-\widetilde{g}_{n}\right)-\left(c_{n}-c_{n+1}\right)}{\left(\widetilde{g}_{n}+c_{n}\right)\left(\widetilde{g}_{n+1}+c_{n+1}\right)}-\frac{1}{f_{3 n}^{2}} \\
& <\frac{\widetilde{g}_{n+1}-\widetilde{g}_{n}}{\left(\widetilde{g}_{n}+c_{n}\right)\left(\widetilde{g}_{n+1}+c_{n+1}\right)}-\frac{1}{f_{3 n}^{2}} \\
& =\frac{B}{\left(\widetilde{g}_{n}+c_{n}\right)\left(\widetilde{g}_{n+1}+c_{n+1}\right) f_{3 n}^{2}},
\end{aligned}
$$

where $B=\left(\widetilde{g}_{n+1}-\widetilde{g}_{n}\right) f_{3 n}^{2}-\left(\widetilde{g}_{n}+c_{n}\right)\left(\widetilde{g}_{n+1}+c_{n+1}\right)$. Now it is enough to show that $B<0$.

By using (5.3), we have

$$
B=A-c_{n} \widetilde{g}_{n+1}-c_{n+1} \widetilde{g}_{n}-c_{n} c_{n+1}<\frac{1024}{81}-c_{n} \widetilde{g}_{n+1} .
$$

Note that

$$
\begin{aligned}
c_{n} \widetilde{g}_{n+1} & =\frac{f_{3 n+3}^{2}-f_{3 n}^{2}-\frac{4}{9}(-1)^{n}}{f_{n}} \\
& >\frac{\left(f_{3 n+3}+f_{3 n}\right)\left(f_{3 n+3}-f_{3 n}\right)}{f_{n}}-\frac{4}{9} \\
& =\frac{\left(f_{3 n+3}+f_{3 n}\right)\left(f_{3 n+2}+f_{3 n-1}\right)}{f_{n}}-\frac{4}{9} \\
& >2\left(f_{3 n+3}+f_{3 n}\right)-1 \geq 19 .
\end{aligned}
$$

It follows that $B<0$.

Remark 5.4 It looks not easy to find the explicit values of $C_{m}$ satisfying (5.1) except for $m=1,3$. By using a computer software program (Maple17 and wolframalpha.com), we found the following. If $m$ is even, then

$$
C_{2} \sim 0.298130320 \ldots
$$




$$
\begin{aligned}
& C_{4} \sim 0.383325938 \ldots \\
& C_{6} \sim 0.397523195 \ldots \\
& C_{8} \sim 0.399637681 \ldots
\end{aligned}
$$

If $m$ is odd, then

$$
\begin{aligned}
& C_{5} \sim 0.406504065 \ldots, \\
& C_{7} \sim 0.400948991 \ldots, \\
& C_{9} \sim 0.400138456 \ldots
\end{aligned}
$$

We might expect that $C_{m}$ tends to $\frac{2}{5}$ as $n \rightarrow \infty$.

\section{Conclusion}

We summarize all the results that have been proved in this paper.

(i) $\left(\sum_{k=n}^{\infty} \frac{1}{f_{k}^{2}}\right)^{-1} \sim f_{n}^{2}-f_{n-1}^{2}+\frac{2}{3}(-1)^{n}$ as $n \rightarrow \infty$,

(ii) $\left(\sum_{k=n}^{\infty} \frac{1}{f_{k} f_{k+2 \ell}}\right)^{-1} \sim f_{n+\ell-1} f_{n+\ell}-\left(f_{\ell}^{2}+(-1)^{\ell}\right) \frac{(-1)^{n}}{3}$ as $n \rightarrow \infty$,

(iii) $\left(\sum_{k=n}^{\infty} \frac{1}{f_{k} f_{k+2 \ell-1}}\right)^{-1} \sim f_{n+\ell-1}^{2}-\left(f_{\ell-1} f_{\ell}+(-1)^{\ell}\right) \frac{(-1)^{n}}{3}$ as $n \rightarrow \infty$,

(iv) $\left(\sum_{k=n}^{\infty} \frac{1}{f_{3 k}^{2}}\right)^{-1} \sim f_{3 n}^{2}-f_{3 n-3}^{2}+\frac{4}{9}(-1)^{n}$ as $n \rightarrow \infty$.

\section{Acknowledgements}

Not applicable.

\section{Funding}

This work was supported by NRF-2018R1D1A1B07050044 from the National Research Foundation of Korea.

\section{Availability of data and materials}

Not applicable.

\section{Competing interests}

The authors declare that they have no competing interests.

\section{Authors' contributions}

All authors read and approved the final manuscript.

\section{Publisher's Note}

Springer Nature remains neutral with regard to jurisdictional claims in published maps and institutional affiliations.

Received: 3 January 2020 Accepted: 24 March 2020 Published online: 01 April 2020

\section{References}

1. Kilic, E., Arikan, T.: More on the infinite sum of reciprocal Fibonacci, Pell and higher order recurrences. Appl. Math. Comput. 219(14), 7783-7788 (2013)

2. Koshy, T.: Fibonacci and Lucas Numbers with Applications, vol. 1, 2nd edn. Wiley, New York (2001)

3. Lee, H.-H., Park, J.-D.: Asymptotic behavior of reciprocal sum of subsequential Fibonacci numbers. Submitted

4. Lin, X.: Partial reciprocal sums of the Mathieu series. J. Inequal. Appl. 2017, Article ID 60 (2017)

5. Liu, R., Wang, A.Y.Z: Sums of products of two reciprocal Fibonacci numbers. Adv. Differ. Equ. 2016, Article ID 136 (2016)

6. Ohtsuka, H., Nakamura, S.: On the sum of reciprocal Fibonacci numbers. Fibonacci Q. 46/47, 153-159 (2008/2009)

7. Ozdemir, G., Simsek, Y.: Generating functions for two-variable polynomials related to a family of Fibonacci type polynomials and numbers. Filomat 30, 969-975 (2016)

8. Ozdemir, G., Simsek, Y.: Identities and relations associated with Lucas and some special sequences. AIP Conf. Proc. 1863, Article ID 300003 (2017). https://doi.org/10.1063/1.4992452

9. Ozdemir, G., Simsek, Y., Milovanović, G.V.: Generating functions for special polynomials and numbers including Apostol-type and Humbert-type polynomials. Mediterr. J. Math. 14(3), Article ID 17 (2017)

10. Vorobiev, N.N.: Fibonacci Numbers. Springer, Basel (2002) 
11. Wang, A.Y.Z., Zhang, F.: The reciprocal sums of even and odd terms in the Fibonacci sequence. J. Inequal. Appl. 2015, Article ID 376 (2015)

12. Zhang, G.J.: The infinite sum of reciprocal of the Fibonacci numbers. J. Math. Res. Expo. 31(6), 1030-1034 (2011)

13. Zhang, W., Wang, T.: The infinite sum of reciprocal Pell numbers. Appl. Math. Comput. 218(10), 6164-6167 (2012)

Submit your manuscript to a SpringerOpen ${ }^{\circ}$ journal and benefit from:

- Convenient online submission

- Rigorous peer review

- Open access: articles freely available online

- High visibility within the field

- Retaining the copyright to your article

Submit your next manuscript at $\gg$ springeropen.com 